\title{
Supporting Information for: Insights into the Stabilization of Fluoride Ion in Ionic Liquids: Pointers to Better Fluorinating Agents
}

\author{
Anjali Gaur, Nikhil V. S. Avula, and Sundaram Balasubramanian \\ Chemistry and Physics of Materials Unit, Jawaharlal Nehru Centre for Advanced Scientific \\ Research, Bangalore 560 064, India
}

\section{Contents}

S1 Calculation of Binding energy in gas phase clusters

S2 Benchmarking using gas-phase quantum calculations of [EMIM]F.EG complexes

S3 Details of Classical MD simulation

S4 Comparison of density of liquid [EMIM]F.EG with experiments

S5 Crystalline Phase

S5.1 Calculations Using Mixed Gaussian and Plane Wave Basis Set . . . . . . . . S10

S5.2 Comparison of interatomic distances in crystalline ([EMIM $] \mathrm{F})_{2}$.EG between X-ray crystallography and DFT calculations . . . . . . . . . . S11

S6 OCCO dihedral angle distribution in EG molecules of liquid [EMIM]F.EGS14 


\section{S1 Calculation of Binding energy in gas phase clusters}

Many different methods have been proposed to calculate the Basis Set Superposition Error (BSSE) for clusters with greater than two fragments, such as VMFC, PAFC, and site-site function counterpoise (SSFC). ${ }^{1}$ Among these, SSFC is computationally the least expensive, and we used this method as implemented in Gaussian 16 to calculate BSSE. Moreover, it has been shown that using a sufficiently large basis set, as used in the current study, reduces BSSE. $^{2}$

For an ion pair of imidazolium chloride ionic liquid, a BSSE of the order of $0.5 \mathrm{~kJ} \mathrm{~mol}^{-1}$ was reported by Hunt's group ${ }^{3}$ using B3LYP-D3/aug-cc-pVTZ level of theory, which is comparable to the level of theory employed in the current study. Moreover, the same group has also shown that BSSE has no effect on the order of relative binding energy values of structures of imidazolium chloride ion pair ionic liquid.

The binding energies were calculated using Equation $\mathrm{S} 1$, where $\Delta E$ is the binding energy of the many-body cluster $i j k \ldots, E(i j k \ldots)$ is the energy of the cluster, and $E\left(i G_{j k l \ldots}\right)$ is the

energy of the $i^{\text {th }}$ fragment in the basis set of $i^{\text {th }}$ fragment and ghost orbitals of all other fragments, except the $i^{\text {th }}$ fragment. ${ }^{4}$

$$
\Delta E(i j k \ldots)=E(i j k \ldots)-\sum_{i} E\left(i G_{k j l \ldots}\right)
$$




\section{S2 Benchmarking using gas-phase quantum calculations of $[$ EMIM]F.EG complexes}

Initial and optimized geometries of several gas-phase [EMIM]F.EG configurations obtained by performing DFT calculation at B3LYP-D3/6-311++G(d,p) level of theory (G16-GP) are shown in table $\mathrm{S} 1$. In all the $[\mathrm{EMIM}] \mathrm{F}$.EG configurations, $\mathrm{EMIM}^{+}$prefers to be in a nonplanar conformation (ethyl chain out of the imidazolium ring plane), even when in some of the initial configurations, $\mathrm{EMIM}^{+}$was arranged to be in coplanar with the ethyl plane. The [EMIM]F.EG configurations can be divided into two types (a) EG in trans conformation present near $\mathrm{H}_{a}$ hydrogen of the $\mathrm{EMIM}^{+}\left(\mathrm{tH}_{a}\right)$ or near $\mathrm{H}_{b}$ hydrogen of the $\mathrm{EMIM}^{+}\left(\mathrm{tH}_{b}\right)$ (b) EG in gauche conformation present near $\mathrm{H}_{a}$ hydrogen of the $\mathrm{EMIM}^{+}\left(\mathrm{gH}_{a}\right)$ or near $\mathrm{H}_{b}$ hydrogen of the $\mathrm{EMIM}^{+}\left(\mathrm{gH}_{b}\right)$. In the type-b configurations, one EG molecule can form two hydrogen bonds with the fluoride ion, while in type-a configurations, it can form only one hydrogen bond with the fluoride ion; hence all the type-b configurations are more stable than type-a configurations. Furthermore, among the respective types, the configuration in which $\mathrm{EG}$ is present near $\mathrm{H}_{a}$ is more stable than the one wherein the $\mathrm{EG}$ is present near $\mathrm{H}_{b}$;

this is due to the fact that the $\mathrm{H}_{a}$ hydrogen is more acidic than the $\mathrm{H}_{b}$ hydrogen. Among the respective $\mathrm{tH}_{a}, \mathrm{tH}_{b}$ and $\mathrm{gH}_{a}$ configurations, the difference in energy of the configurations when EG is present near the ethyl chain, and when EG is present near the methyl chain is less than $5 \mathrm{~kJ} / \mathrm{mol}$. In $\mathrm{gH}_{b}$ configurations, no stable configuration was found in which EG could be present near the methyl group, the configurations that were initialized with such an arrangement changed to $\mathrm{gH}_{a}$ configuration after the optimization. 
Table S1: Initial and geometry optimized structures of different configurations of [EMIM]F.EG complex. Structures were optimized at B3LYP-D3/6-311++G(d,p) level of theory. OCCO dihedral angle is mentioned in bracket. Color code: hydrogen, white; carbon, gray; nitrogen, blue; oxygen, red; fluorine, salmon.

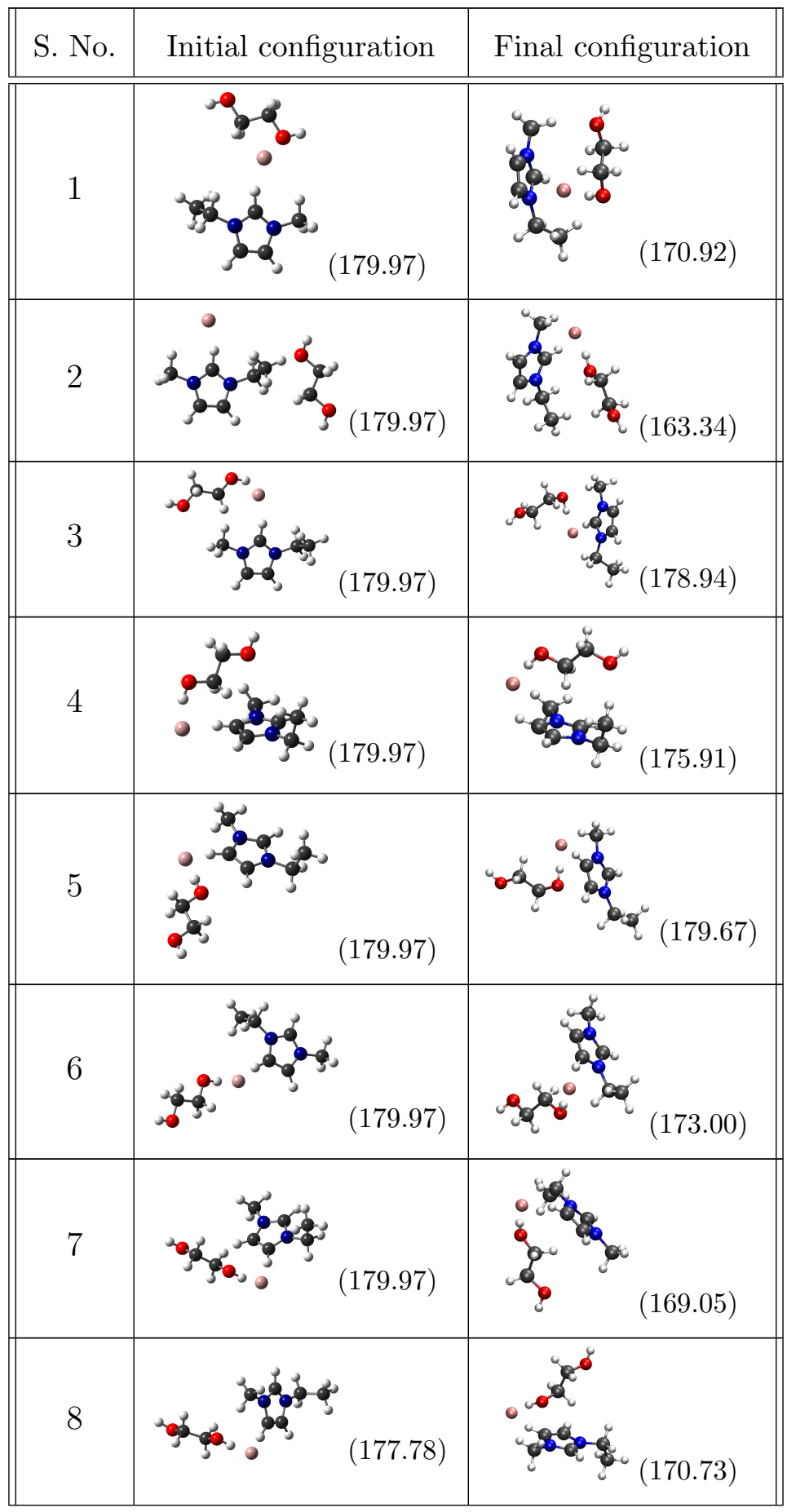


Table S1: Initial and geometry optimized structures of different configurations of [EMIM]F.EG complex. Structures were optimized at B3LYP-D3/6-311++G(d,p) level of theory. OCCO dihedral angle is mentioned in bracket. Color code: hydrogen, white; carbon, gray; nitrogen, blue; oxygen, red; fluorine, salmon.

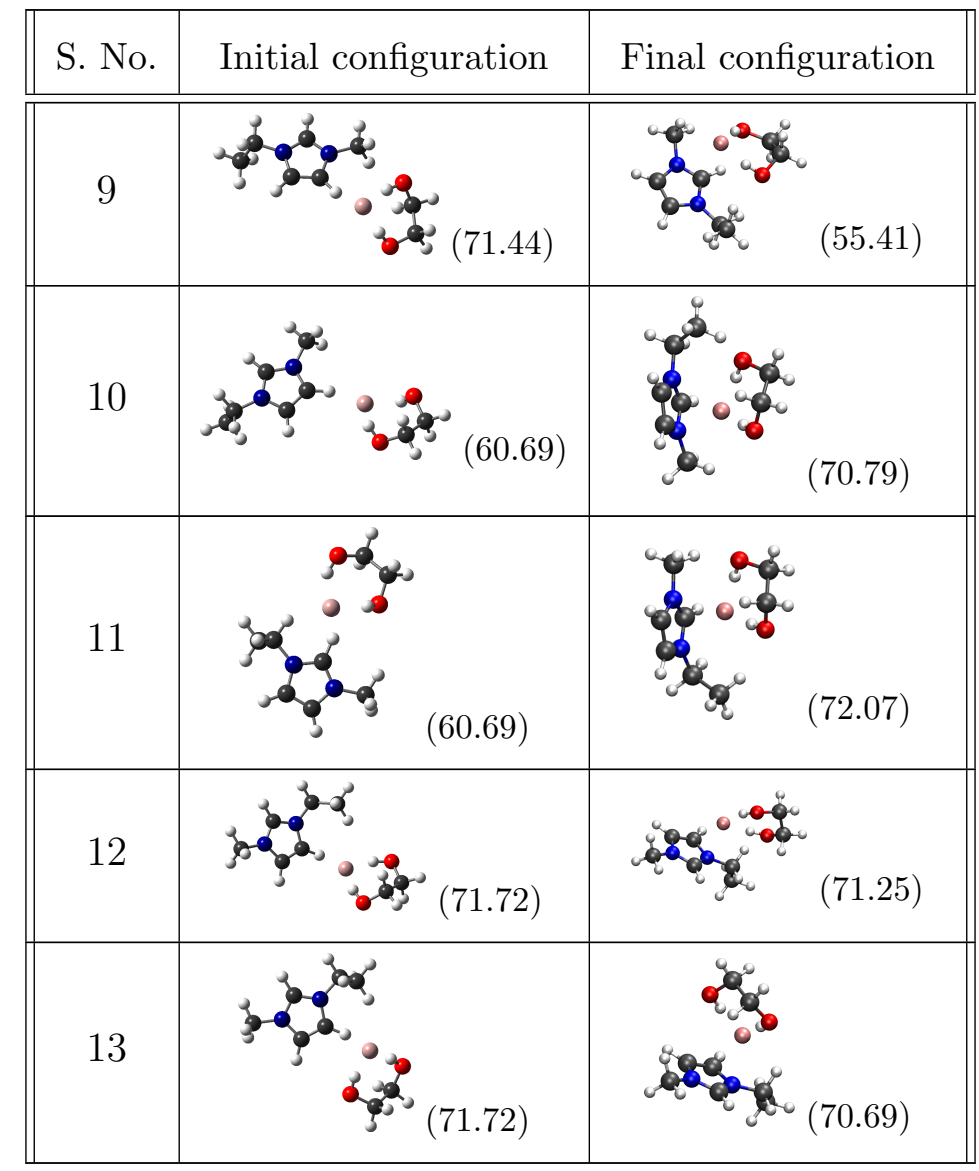

In order to perform periodic DFT calculation for the crystalline and liquid phase using a mixed basis set (CP2K-PBC), the selection of basis set and XC functional demands consideration of accuracy and computational cost of the calculation. ${ }^{5-8}$ Therefore, we performed gas-phase quantum calculations using two different mixed basis sets for thirteen different configurations of the [EMIM]F.EG complex (CP2K-GP), which are commonly used for condensed phases - TZV2P and DZVP-MOLOPT-SR, combined with the PBE XC functional. We benchmarked these CP2K-GP calculations by comparing the relative energies and final optimized geometries of several gas-phase [EMIM-F].EG configurations against the respec- 
tive G16-GP calculations. The geometries obtained from CP2K-GP calculations matched very closely with the geometries obtained from G16-GP (Figure S1) calculations. Figure S1 shows relative energies of all the configurations obtained from different quantum calculations. The relative energies of the configurations obtained from both the CP2K-GP calculations matched closely with those obtained using G16-GP calculation. However, the DZVP-MOLOPT-SR basis set is computationally cheaper to use than TZV2P; hence DZVPMOLOPT-SR basis set is chosen to perform CP2K-PBC calculations for the systems studied here.

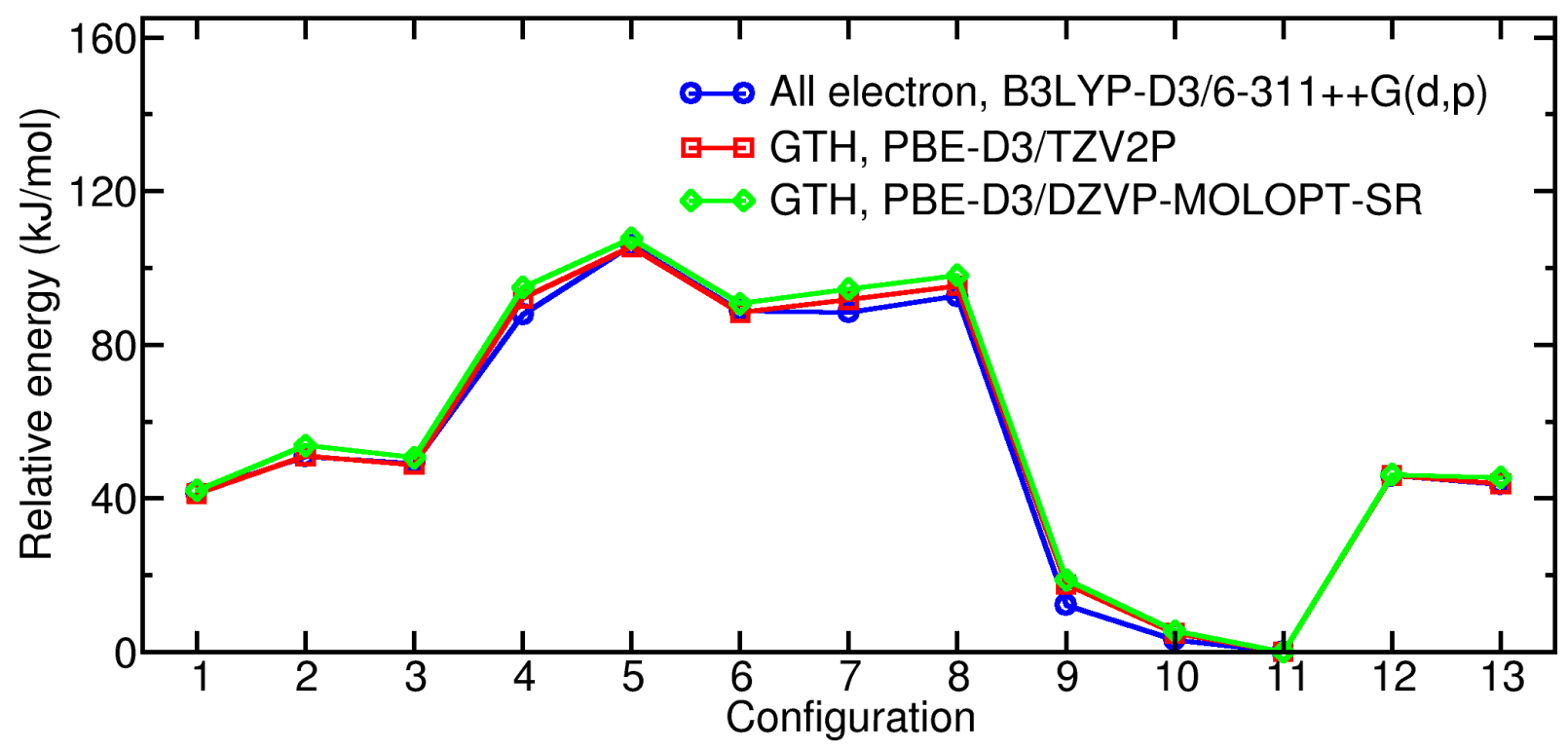

Figure S1: Relative energies of configurations of [EMIM]F-EG complexes geometry optimized in the gas-phase at different levels of theory.

\section{S3 Details of Classical MD simulation}

Classical MD simulations of $[\mathrm{EMIM}] \mathrm{F} . \mathrm{xEG}(\mathrm{x}=0.5,1,1.5)$ liquids were performed using Gromacs-2018.3. ${ }^{9}$ The initial configurations of molecules in the liquid were generated using PACKMOL. ${ }^{10}$ Table S2 lists the number of molecules and the length of the cubic box of all the systems. The force field parameters for $[\mathrm{EMIM}]^{+}$were taken from the work of Kelkar et al. ${ }^{11}$ and that for EG were taken from the work of Kaur and Kashyap ${ }^{12}$ and for $\mathrm{F}^{-}$, 
AMBER $^{13}$ parameters were used. Three dimensional periodic boundary conditions were applied. The systems were first energy minimized using the steepest descent ${ }^{14}$ algorithm. The equations of motion were integrated using the leap-frog algorithm with a time step of 1 fs. As the box dimensions were relatively small (in view of the expensive nature of BOMD simulation for which these classical MD simulations were a prerequisite), the interaction cutoff was chosen to be half the box length and not as per the prescription of each of the force fields. Long-range Coulombic interactions were calculated using Particle-Mesh Ewald (PME) solver. Long-range correction to the energy and pressure was applied. Subsequently, isothermal-isobaric simulations were performed at different temperatures and 1 bar for $20 \mathrm{~ns}$ to obtain the equilibrium density of the systems. All covalent bonds involving hydrogen atoms were constrained using the LINCS algorithm. Nosé-Hoover thermostat ${ }^{15,16}$ with a coupling constant of 1 ps and Parrinello-Rahman barostat ${ }^{17}$ with a coupling constant of 10 ps were employed to control temperature and pressure, respectively.

Table S2: Details of MD simulations of [EMIM]F.xEG $(\mathrm{x}=0.5,1,1.5)$ systems.

\begin{tabular}{||c|c|c|c||}
\hline System & No. of [EMIM]F pairs & No. of EG molecules & Box length $(\AA)$ \\
\hline \hline [EMIM]F.0.5EG & 40 & 20 & 21.259 \\
[EMIM]F.EG & 26 & 26 & 19.436 \\
[EMIM]F.1.5EG & 26 & 39 & 20.362 \\
\hline
\end{tabular}




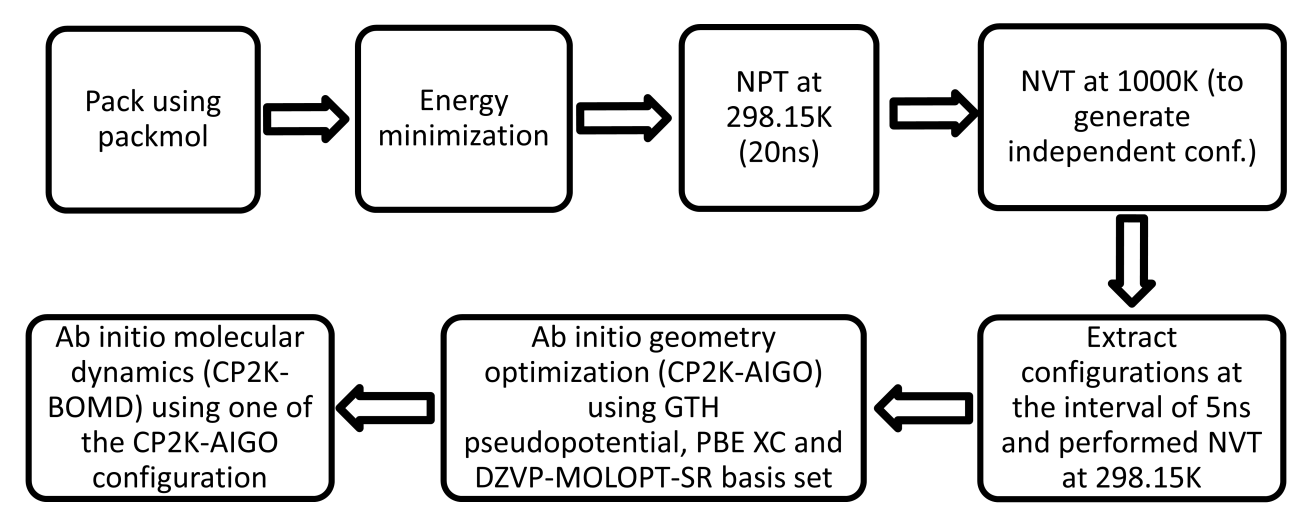

Figure S2: Flow diagram of the procedure that was followed to generate liquid snapshots and to perform CP2K-AIGO calculations. Ten independent liquid phase snapshots obtained from classical MD simulations were taken up for further CP2K-AIGO. One of the post CP2K-AIGO configurations was used as the initial configuration for the CP2K-BOMD run.

\section{S4 Comparison of density of liquid [EMIM]F.EG with experiments}

Figure S3 exhibits the experimental and calculated densities at different temperatures. The liquid was simulated at different temperatures for $20 \mathrm{~ns}$ out of which the last $10 \mathrm{~ns}$ was used to compute equilibrium density. A maximum deviation of $1.2 \%$ with respect to the experiment is observed. The force field closely reproduces the density of the simulated system. 


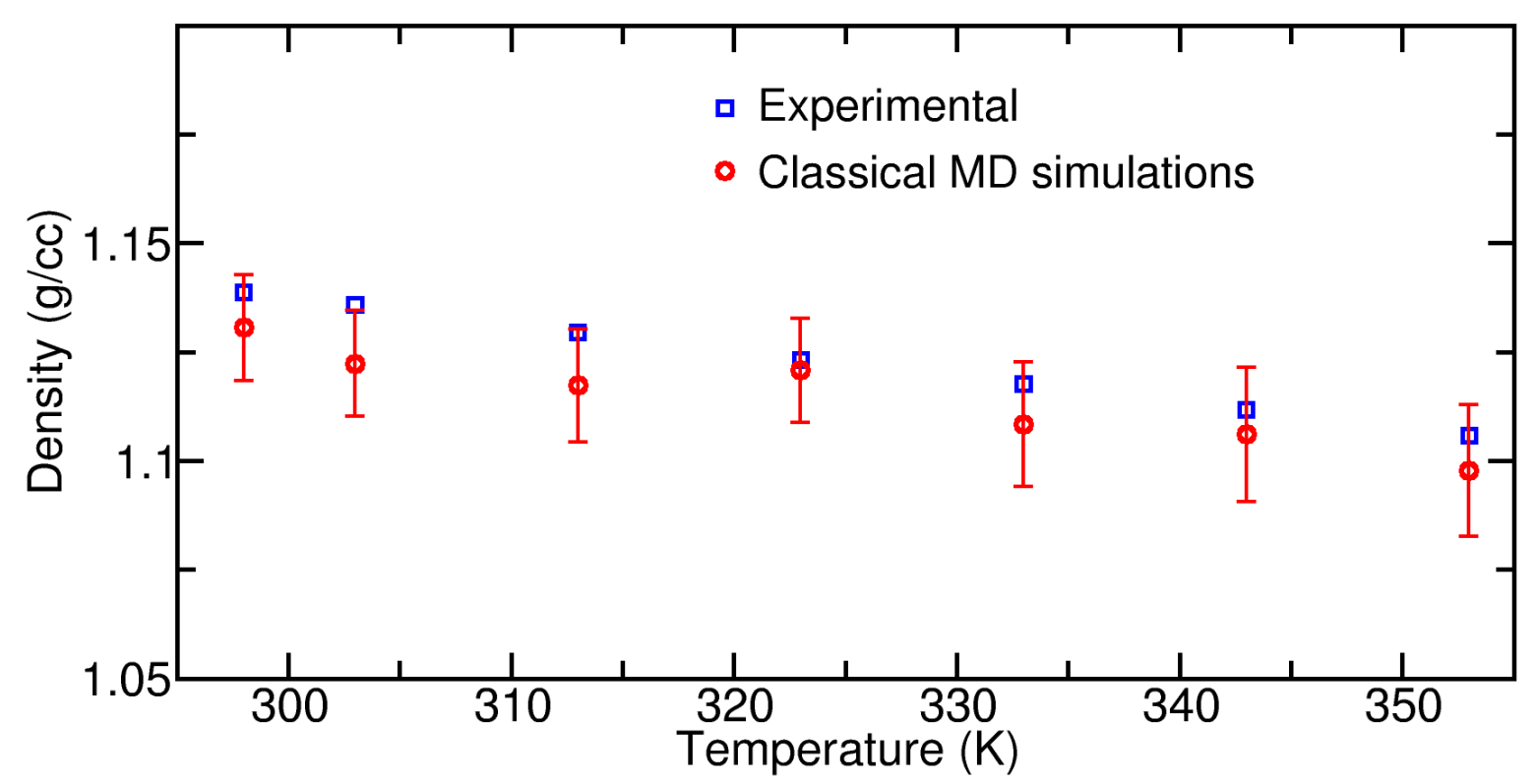

Figure S3: Experimental (blue) and calculated density (red) for liquid [EMIM]F.EG at various temperatures, the deviation ranges from $0.2 \%$ to $1.2 \%$.

\section{S5 Crystalline Phase}

\section{S5.1 Calculations Using Mixed Gaussian and Plane Wave Basis Set}

Apart from benchmarking against G16-GP quantum chemical calculations of complexes, the PBE functional based approach can also be tested against experimental results on the crystalline phase of $([\mathrm{EMIM}] \mathrm{F})_{2} . \mathrm{EG}$, whose crystal structure was reported experimentally. Thus, periodic DFT calculations were carried out using CP2K software. The size of the simulation cell considered for the crystal was $1 \times 1 \times 1$. Several combinations of DZVPMOLOPT-SR, TZV2P basis set, PBE, B3LYP, PBE0 XC functionals, and rVV10, Grimme's D3 dispersion corrections were used to find the most satisfactory combination. Other details of the calculations are the same as discussed for the gas-phase system.

Geometry optimization of the crystal was performed using the DZVP-MOLOPT-SR basis set and PBE XC functional. The experimentally reported crystal structure is shown in 
Figures S4 and S5. Post geometry optimization, some of the covalent bond distances involving hydrogen atoms as well as hydrogen bond distances deviated by 10 to $25 \%$ from the experimentally reported distances (see Table S3), which was resolved to arise from the indeterminacy of hydrogen atom locations in experiment, due to its weak scattering of X-rays.

\section{S5.2 Comparison of interatomic distances in crystalline $([\mathrm{EMIM}] \mathrm{F})_{2}$.EG between X-ray crystallography and DFT calculations}

Figure S4 shows the experimentally reported crystal structure of $([\mathrm{EMIM}] \mathrm{F})_{2}$.EG in two different orientations. ${ }^{18}$ In the crystalline phase, the ratio of [EMIM]F to EG is $2: 1$, and wherein three EMIM cations and one EG surround one fluoride ion. The orientation of each EMIM cation around the fluoride ion is different, each forming hydrogen bond through $\mathrm{H}_{a}$, $\mathrm{H}_{b}$, and $\mathrm{H}_{c}$, respectively, as shown in Figure S5. EG is in the trans conformation, and one EG molecule forms hydrogen bonds with two fluoride ions.
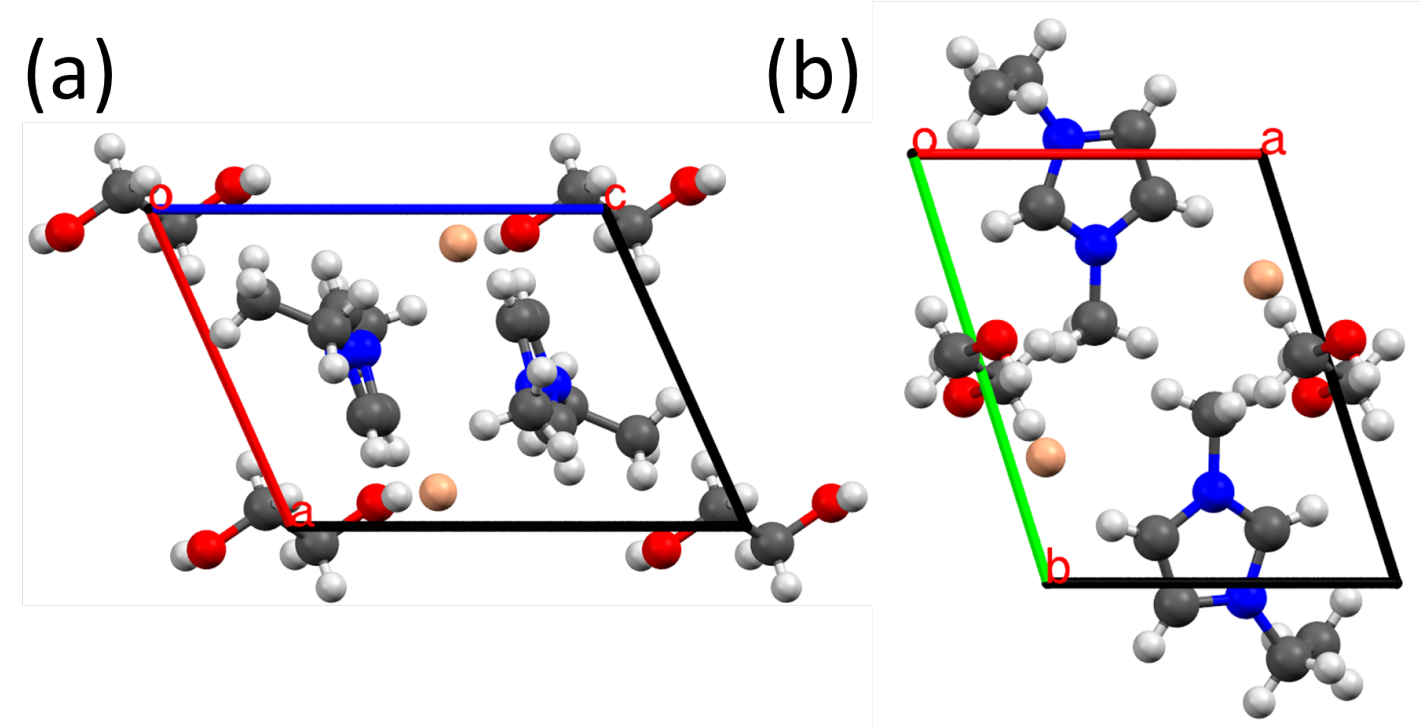

Figure S4: Experimentally determined crystal structure of $([\mathrm{EMIM}] \mathrm{F})_{2}$.EG (a) projection into ac plane (b) projection into ab plane. Color code: hydrogen, white; carbon, gray; nitrogen, blue; oxygen, red; fluorine, salmon 


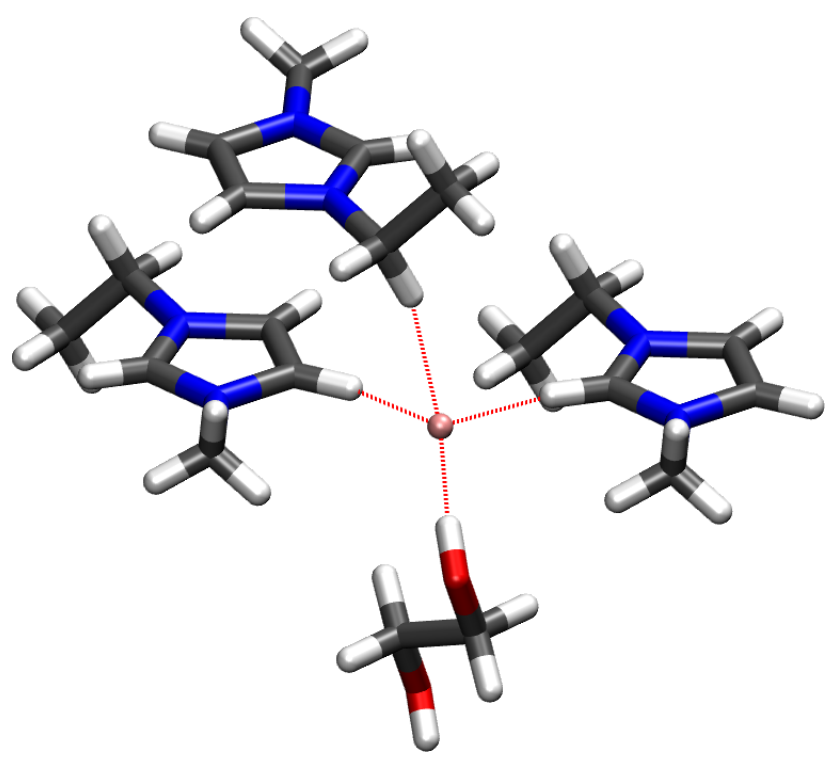

Figure S5: Coordination environment of fluoride in the $([\mathrm{EMIM}] \mathrm{F})_{2}$.EG crystal system. Color code: hydrogen, white; carbon, gray; nitrogen, blue; oxygen, red; fluorine, salmon

Table S3: Hydrogen bond and a few intramolecular covalent bond distances obtained post geometry optimization (CP2K-AIGO) of the crystal, performed using the DZVP-MOLOPT$\mathrm{SR}$ basis set and PBE XC functional. The difference between experimental and calculated distances involving hydrogen atom ranges from $\sim 10$ to $20 \%$, while that involving heavy atoms are within $2 \%$.

\begin{tabular}{||c|c|c||}
\hline \multirow{2}{*}{ Bond } & \multicolumn{2}{|c|}{ Bond Distance $(\AA)$} \\
\cline { 2 - 3 } & Experimental & Calculated \\
\hline \hline $\mathrm{OG}-\mathrm{HO}$ & 0.82 & 1.03 \\
$\mathrm{C}_{2}-\mathrm{H}_{a}$ & 0.93 & 1.11 \\
$\mathrm{OH}-\mathrm{F}$ & 1.67 & 1.49 \\
$\mathrm{H}_{a}-\mathrm{F}$ & 1.89 & 1.69 \\
$\mathrm{C}_{2}-\mathrm{F}$ & 2.80 & 2.80 \\
$\mathrm{~N}_{1}-\mathrm{C}_{2}$ & 1.33 & 1.35 \\
$\mathrm{~N}_{3}-\mathrm{C}_{4}$ & 1.38 & 1.39 \\
$\mathrm{C}_{4}-\mathrm{C}_{5}$ & 1.35 & 1.37 \\
$\mathrm{OG}-\mathrm{F}$ & 2.48 & 2.52 \\
$\mathrm{CG}-\mathrm{CG}$ & 1.50 & 1.54 \\
$\mathrm{CG}-\mathrm{OG}$ & 1.41 & 1.43 \\
\hline
\end{tabular}

In order to resolve the difference between experiment and calculations observed for distances involving hydrogen atom, we repeated the same calculation using the non-hybrid functional BLYP, as well as the hybrid functional PBE0 along with the rVV10 non-local cor- 
relation functional. ${ }^{19,20}$ All the calculations yield approximately similar distances as before, and these values are listed in Table S4. Note that the experimental values were reported using X-ray crystallography and the discrepancies between experiments and our results are thus likely due to the inability of the X-ray diffraction method to locate the hydrogen atom precisely.

Table S4: Hydrogen bond and a few intramolecular bond distances calculated using several XC functionals viz., PBE, BLYP and the hybrid functional PBE0 combined with rVV10 and Grimme-D3 dispersion correction. All these methods yield more or less similar results.

\begin{tabular}{||c|c|c|c|c|c|c||}
\hline \multirow{2}{*}{ Bond } & \multirow{2}{*}{$\begin{array}{c}\text { Experimental } \\
\text { bond }\end{array}$} & \multicolumn{5}{|c||}{ Calculated bond distance $(\AA)$} \\
\cline { 3 - 7 } & distance $(\AA)$ & \multicolumn{3}{|c|}{ DZVP-MOLOPT-SR } & \multicolumn{2}{c||}{ TZV2P } \\
\cline { 3 - 7 } & 0.82 & 1.01 & 1.01 & 1.01 & 1.02 & 1.02 \\
\hline \hline OG-HO & 1.10 & 1.10 & 1.10 & 1.10 & 1.10 \\
$\mathrm{C}_{2}-\mathrm{H}_{a}$ & 0.93 & 1.50 & 1.51 & 1.50 & 1.52 & 1.53 \\
$\mathrm{OH}-\mathrm{F}$ & 1.67 & 1.70 & 1.68 & 1.69 & 1.74 & 1.71 \\
$\mathrm{Ha}-\mathrm{F}$ & 1.89 & 2.80 & 2.84 & 2.80 & 2.84 & 2.81 \\
$\mathrm{C}_{2}-\mathrm{F}$ & 2.80 & 1.35 & 1.33 & 1.33 & 1.35 & 1.35 \\
$\mathrm{~N}_{1}-\mathrm{C}_{2}$ & 1.33 & 1.39 & 1.38 & 1.38 & 1.40 & 1.39 \\
$\mathrm{~N}_{3}-\mathrm{C}_{4}$ & 1.38 & 1.37 & 1.35 & 1.36 & 1.37 & 1.37 \\
$\mathrm{C}_{4}-\mathrm{C}_{5}$ & 1.35 & 2.51 & 2.52 & 2.52 & 2.54 & 2.55 \\
$\mathrm{OG}-\mathrm{F}$ & 2.48 & 1.54 & 1.52 & 1.52 & 1.54 & 1.54 \\
$\mathrm{CG}-\mathrm{CG}$ & 1.50 & 1.43 & 1.41 & 1.41 & 1.44 & 1.44 \\
$\mathrm{CG}-\mathrm{OG}$ & 1.41 & & & & &
\end{tabular}

Moreover, the OG-HO covalent bond distance in neat EG in its gas-phase is $0.92 \AA$, while in its crystalline phase, it is $1.00 \AA$, which is a $9 \%$ increase. ${ }^{21}$ In crystalline EG, there is strong intermolecular hydrogen bonding, which increases the OG-HO covalent bond distance. Thus, the OG-HO distance that we obtained in our calculations of $1.03 \AA$ for crystalline $([\mathrm{EMIM}] \mathrm{F})_{2}$.EG is in good agreement with that observed in neutron scattering experiments of crystalline EG. Therefore, the value of $0.82 \AA$ reported in the experimental crystal structure of $([\mathrm{EMIM}] \mathrm{F})_{2}$.EG is unreasonable. Hence the calculated OG-HO covalent distance of $1.03 \AA$ should be the correct value.

These calculations affirm that the procedures employed in the calculations are robust and can be deployed for simulations of the liquid state. 


\section{S6 OCCO dihedral angle distribution in EG molecules of liquid [EMIM]F.EG}

The OCCO dihedral angle distribution in pure EG liquid has been studied extensively through experiments as well as simulations. Both experimental and computational studies concur that the central OCCO dihedral bond adopts the gauche (60 \pm 20 degrees) conformation and it is the most populated angle in the liquid state. ${ }^{22-27}$ The classical MD simulations of [EMIM]F.EG liquid yielded the highest populationof EG molecules to be in the cis (0 degrees) conformation. However, during geometry optimization (CP2K-AIGO), the population of the cis conformation reduced to zero, while that of the gauche conformation (60 degrees) increased (Figure S6), in agreement with experiments.

The difference in OCCO dihedral distribution in EG molecules obtained from CP2KAIGO and classical MD was indeed surprising on two counts - firstly, that the dihedral preferences in the force field and that from quantum DFT were different and secondly, that just a simple geometry optimization of a complex fluid was able to change the dihedral angle distribution so drastically. 


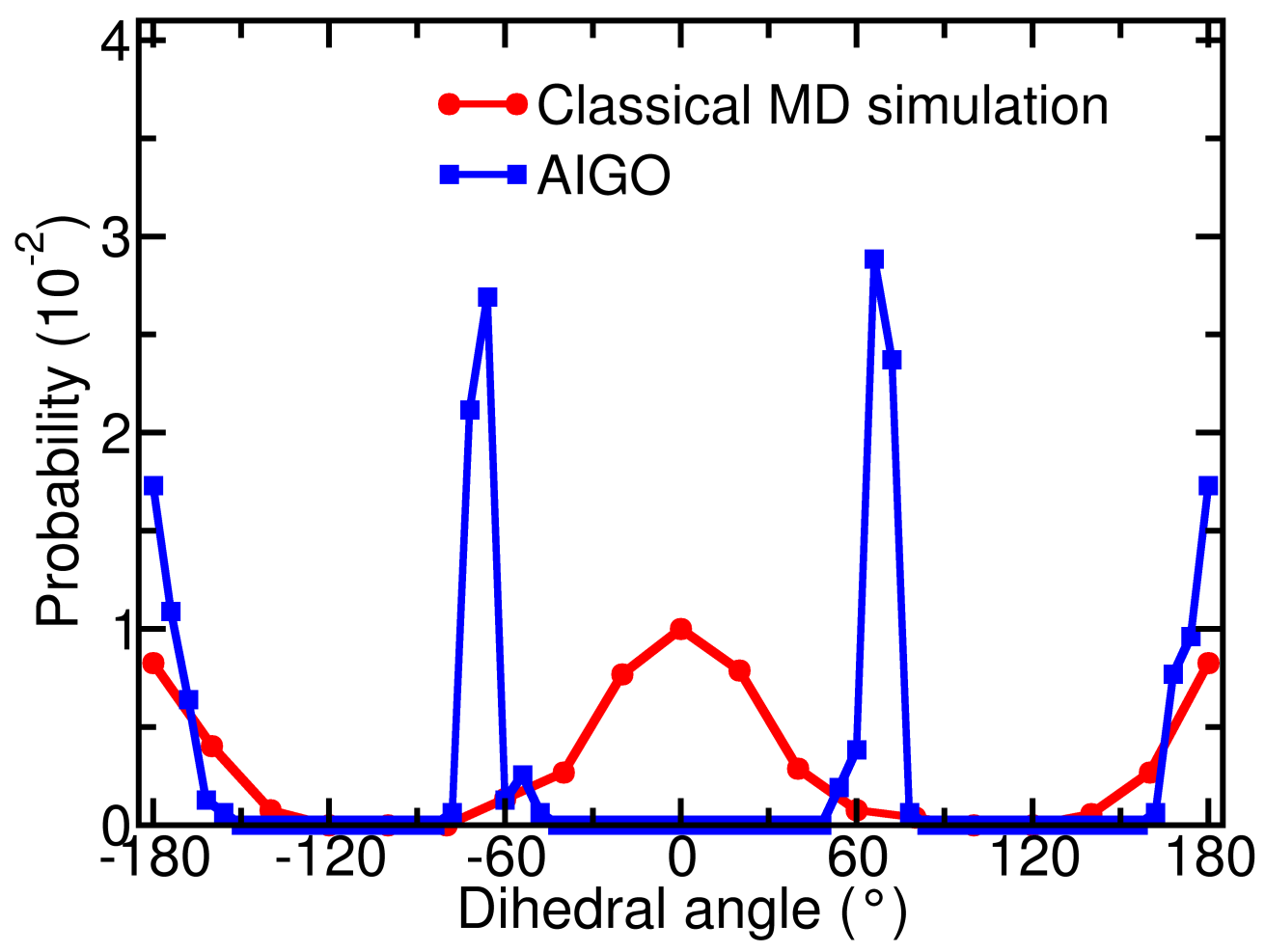

Figure S6: Probability density $(\mathrm{P}(\mathrm{n}))$ distribution of OCCO dihedral angle of EG molecules in liquid [EMIM]F.EG for snapshots obtained from classical MD simulation and post their geometry optimization within a quantum DFT framework.

To understand the disparity between the dihedral distributions of EG from classical MD and CP2K-AIGO, we performed relaxed dihedral scans of a single molecule of EG, using quantum chemical methods and compared them with the dihedral potential energy function prescribed in the force field of Ref 12. Figure S7 shows the relative energy of EG molecule with respect to the OCCO dihedral angle, calculated using these procedures.

According to the dihedral potential energy function of the force field for EG, ${ }^{12}$ the cis conformer is the most stable while it is the least stable according to quantum dihedral potential energy scans. Quantum calculations show that the gauche conformer is the most stable and the energy difference between gauche and cis conformers is $25 \mathrm{~kJ} / \mathrm{mol}$. Quantum potential energy dihedral scan performed using atom-centered basis set (and with all-electron) and that performed with mixed basis set are in close agreement with each other.

As discussed in the section on gas-phase calculations, EG can form bidentate hydrogen 


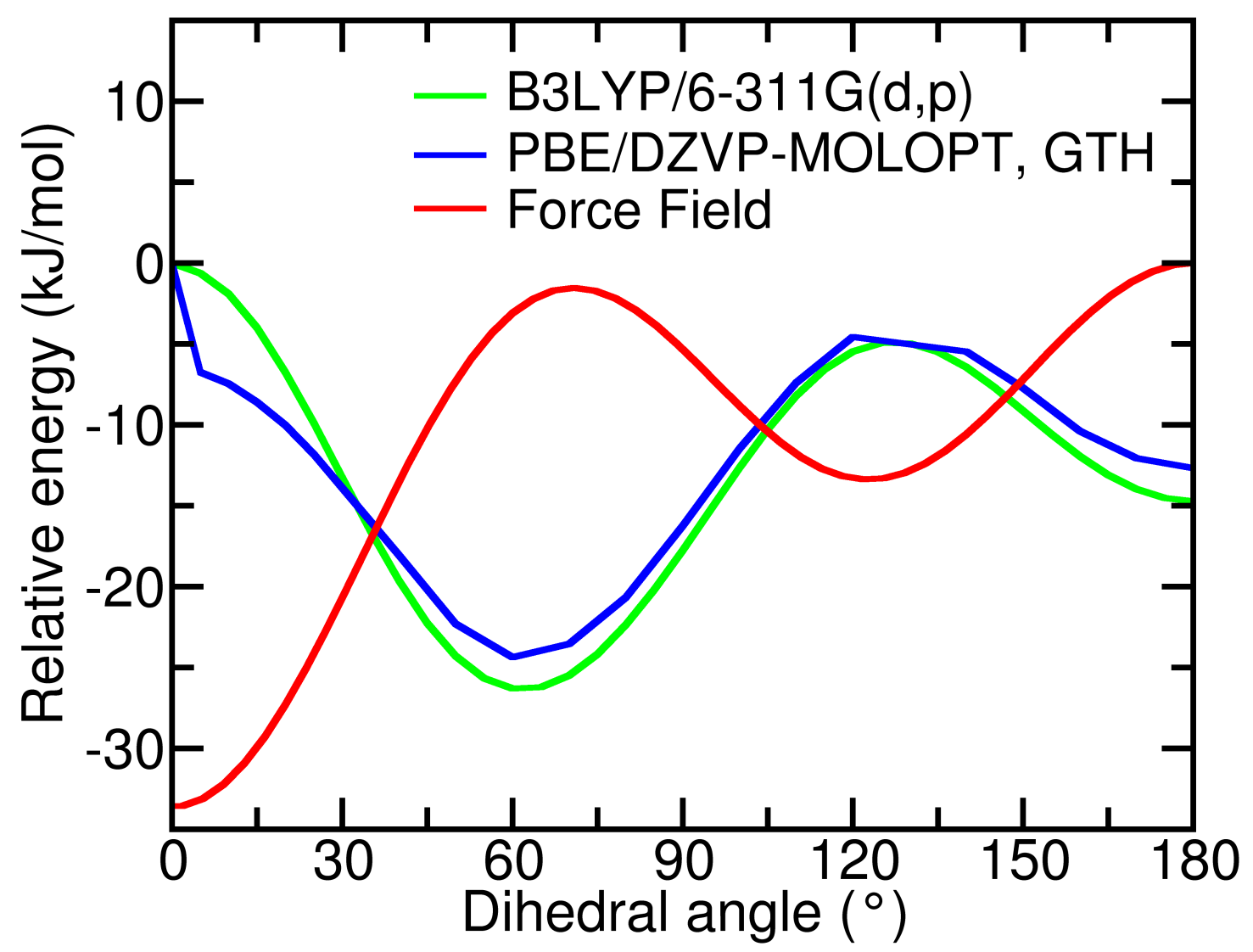

Figure S7: Profile of relaxed potential energy scans of the OCCO dihedral angle of EG molecule in gas-phase, carried out at two different levels of quantum theory and the dihedral potential energy function prescribed in the force field of Ref. 12. The relative stability of different conformers of EG as described by the force field ${ }^{12}$ does not agree with our quantum calculations.

bonds with one fluoride ion in its gauche form, providing extra stabilization to the gauche conformer. Coupled with the deep well in the dihedral potential energy for the gauche conformation, this intermolecular interaction further explains the large peak for this conformer in the dihedral angle distribution (see Figure S6) obtained from CP2K-AIGO. The secondary peak in the distribution seen for the trans conformation is in agreement with the second basin found in the dihedral potential energy scan for that conformer.

The observed difference between the classical MD and CP2K-AIGO in terms of OCCO dihedral distribution in liquid [EMIM]F.EG is thus due to the incorrect dihedral parameters prescribed in the force field of Reference 12. Therefore, the force field parameters for EG 
need to be modified. The refinement of the dihedral potential parameters for EG will be taken up in a subsequent work.

Our focus here, however, is in quantum modeling of liquid [EMIM]F.EG. Given that a simple geometry optimization within DFT itself is able to change the dihedral distributions and that the CP2K-BOMD simulations (vide infra) were initiated from configurations which underwent the CP2K-AIGO procedure, the incorrect distribution in the classical MD simulations, arising from the force field, need not concern us.

\section{S7 Hydrogen bonding}

The radial distribution function between fluoride and $\mathrm{H}_{a}$ atom as well as the distribution of the $\mathrm{C}_{2}-\mathrm{H}_{a}-\mathrm{F}$ angle calculated from liquid snapshots before and after ab initio geometry optimization are shown in Figure S8. To calculate the angle distribution, fluorides around $\mathrm{H}_{a}$ atoms within a cutoff distance were first identified, and later the angle between the neighboring fluorides, $\mathrm{H}_{a}$, and $\mathrm{C}_{2}$ was calculated. The distance cutoff was taken to be the position of the first minimum of $\mathrm{F}-\mathrm{H}_{a}$ pair correlation function, which is $2.31 \AA$. Compared to the distributions obtained from classical MD simulation, we observe that the hydrogen bond distance of $\mathrm{F}-\mathrm{H}_{a}$ obtained from $\mathrm{CP} 2 \mathrm{~K}-\mathrm{AIGO}$ shifts to lower distances by about $0.21 \AA$, and the hydrogen bond angle too became more linear. Together, these two effects suggest stronger hydrogen bonding between the cation and the fluoride anion in the DFT representation relative to that in the force field.

$\mathrm{F}-\mathrm{HO}$ is another important hydrogen bond which stabilizes the fluoride in [EMIM]F.EG ionic liquid. The F-HO radial distribution function and OG-HO-F angle distribution functions are shown in Figure S9. The angle distribution is calculated in the same fashion as before. The distance cutoff was taken to be the position of the first minimum of the FHO pair correlation function, which is $1.82 \AA$. There is no significant change in the $\mathrm{F}-\mathrm{HO}$ hydrogen bond geometry, post CP2K-AIGO. 

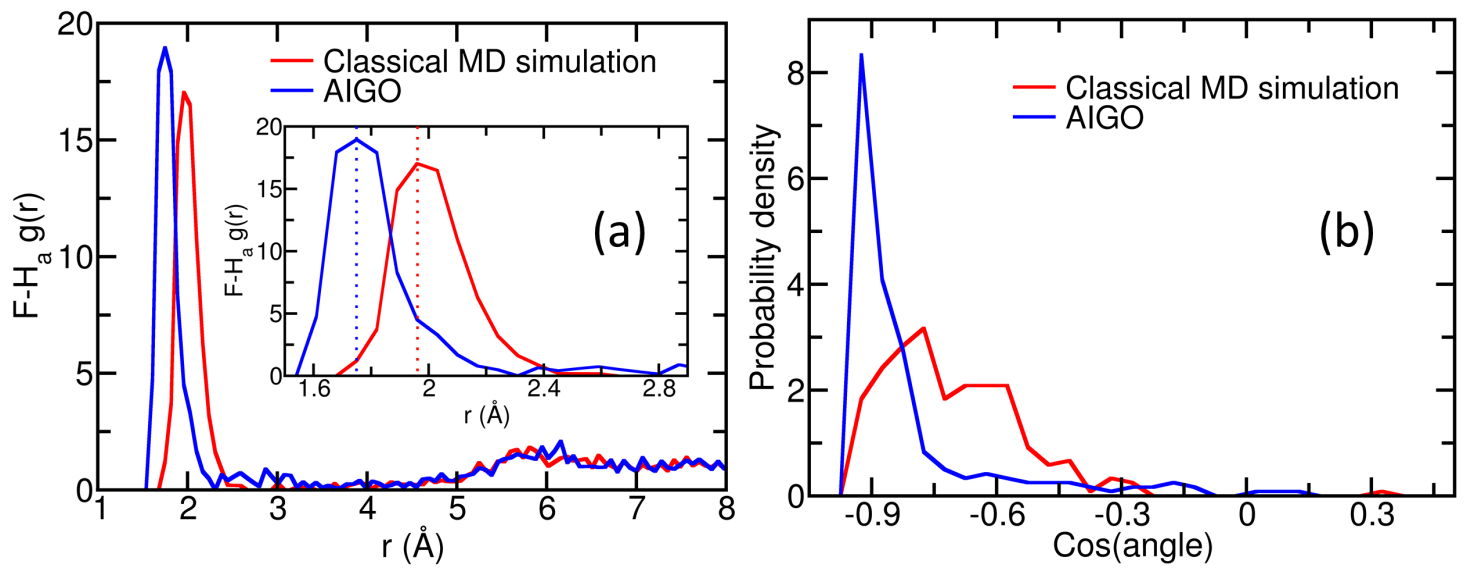

Figure S8: (a) RDF between fluoride and $\mathrm{H}_{a}$ atoms and (b) $\mathrm{C}_{2}-\mathrm{H}_{a}-\mathrm{F}$ angle distribution obtained from classical MD and CP2K-AIGO for [EMIM]F.EG liquid. The $\mathrm{F}_{-} \mathrm{H}_{a}$ rdf peak shifted to lower $\mathrm{F}-\mathrm{H}_{a}$ distance by $0.21 \AA$, post CP2K-AIGO. Further, the $\mathrm{C}_{2}-\mathrm{H}_{a}-\mathrm{F}$ angles become more linear.
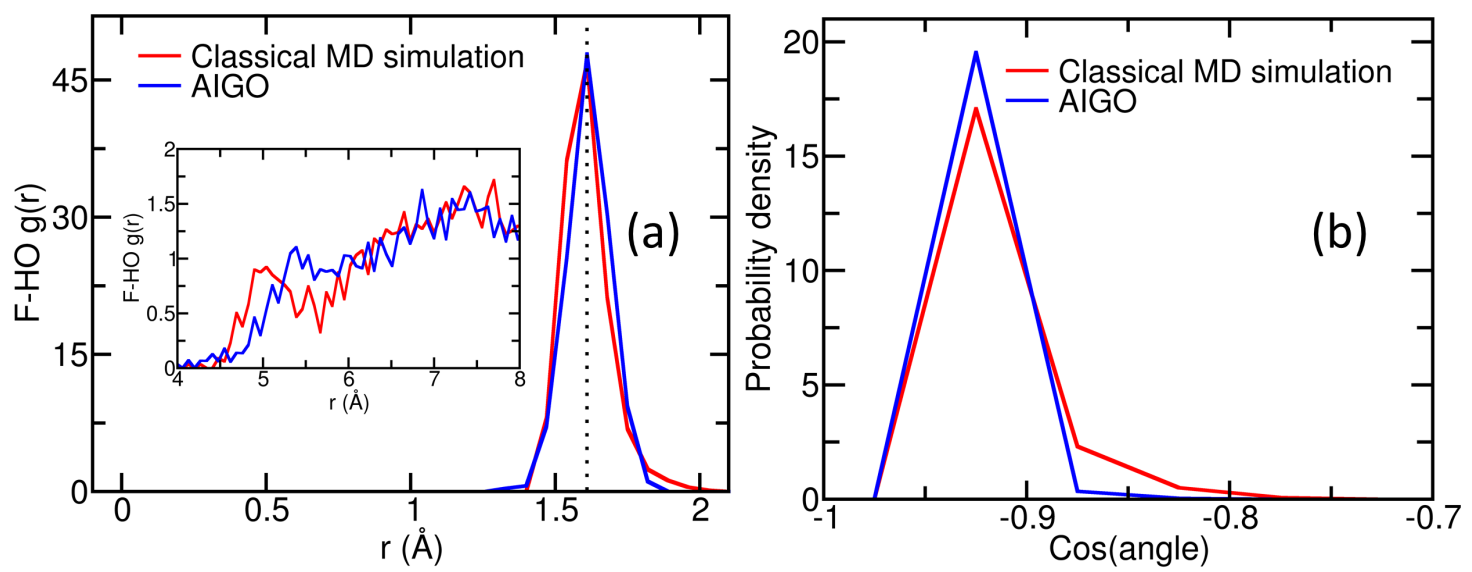

Figure S9: (a) RDF between fluoride and $\mathrm{HO}$ atoms and (b) OG-HO-F angle distribution obtained from classical MD and CP2K-AIGO for [EMIM]F.EG system. There is no significant change in F-HO RDF peak position and the OG-HO-F angle distribution, post CP2K-AIGO. 


\title{
S8 Coordination environment of some selected
}

\author{
fluoride ions
}
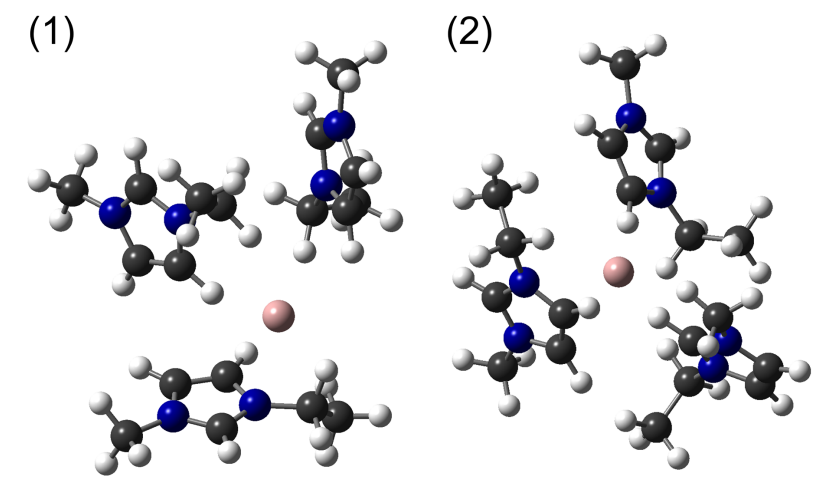

(3)

(4)
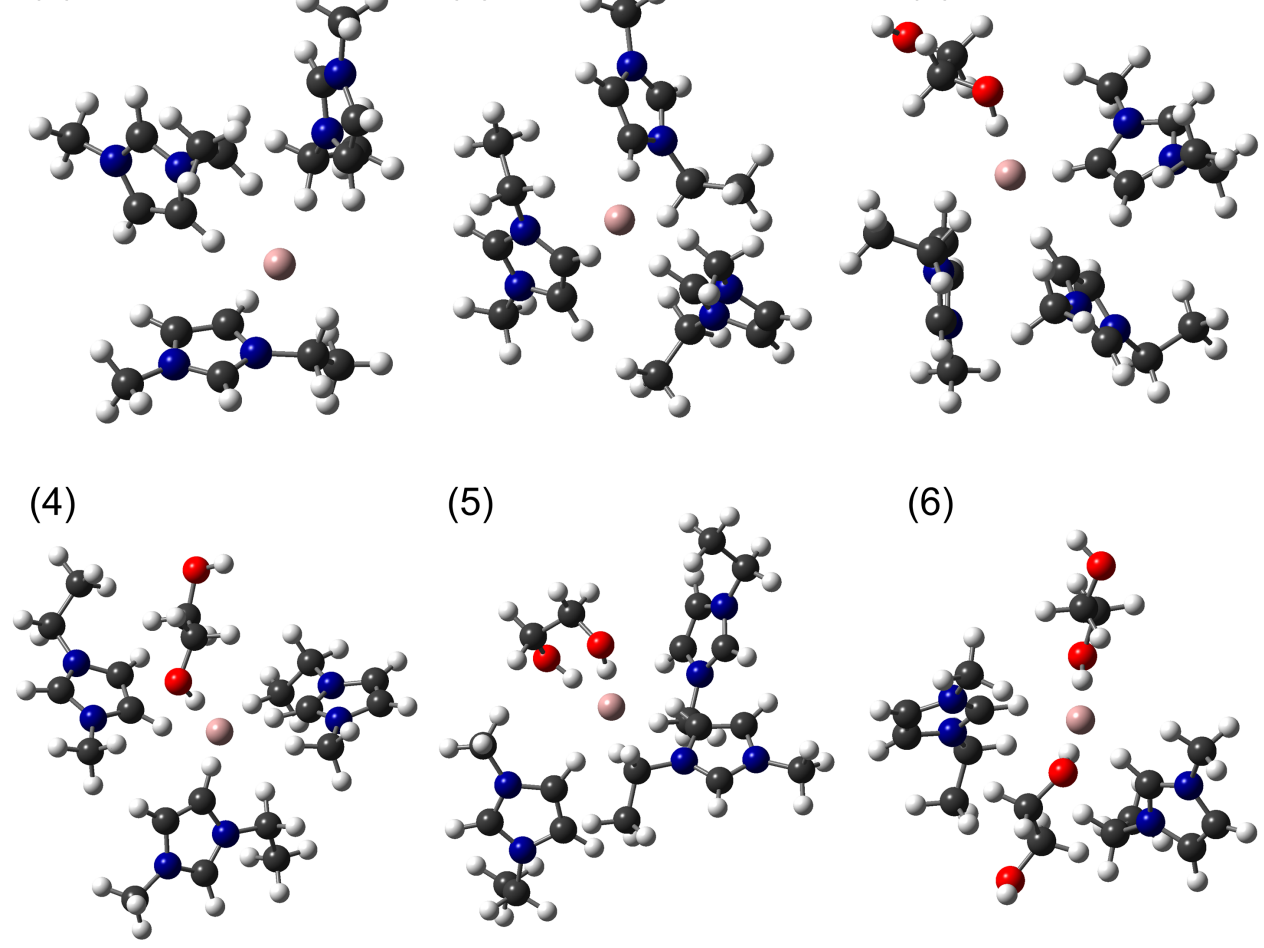

(5)

(7)

(8)
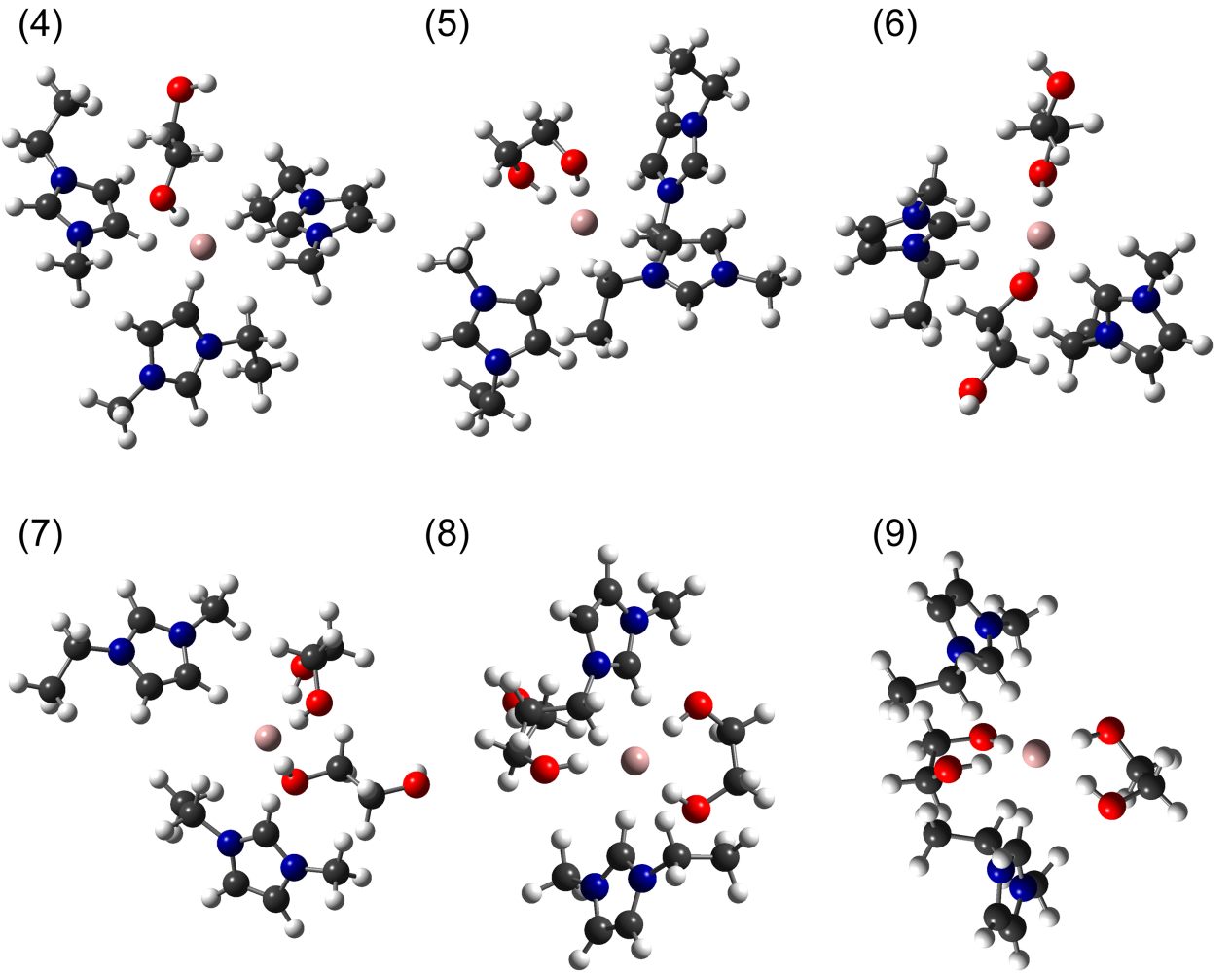

(9)

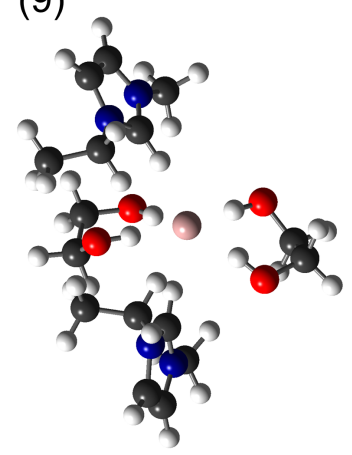

Figure S10: Coordination environment of some selected fluoride ions obtained post CP2KAIGO calculations of liquid [EMIM]F.EG. Molecules, whose any atom is present within a distance of 2.5 of the fluoride ion, are shown. These type of clusters were used to calculate binding energy values shown in Table 4. Color code: hydrogen, white; carbon, gray; nitrogen, blue; oxygen, red; fluorine, salmon. 

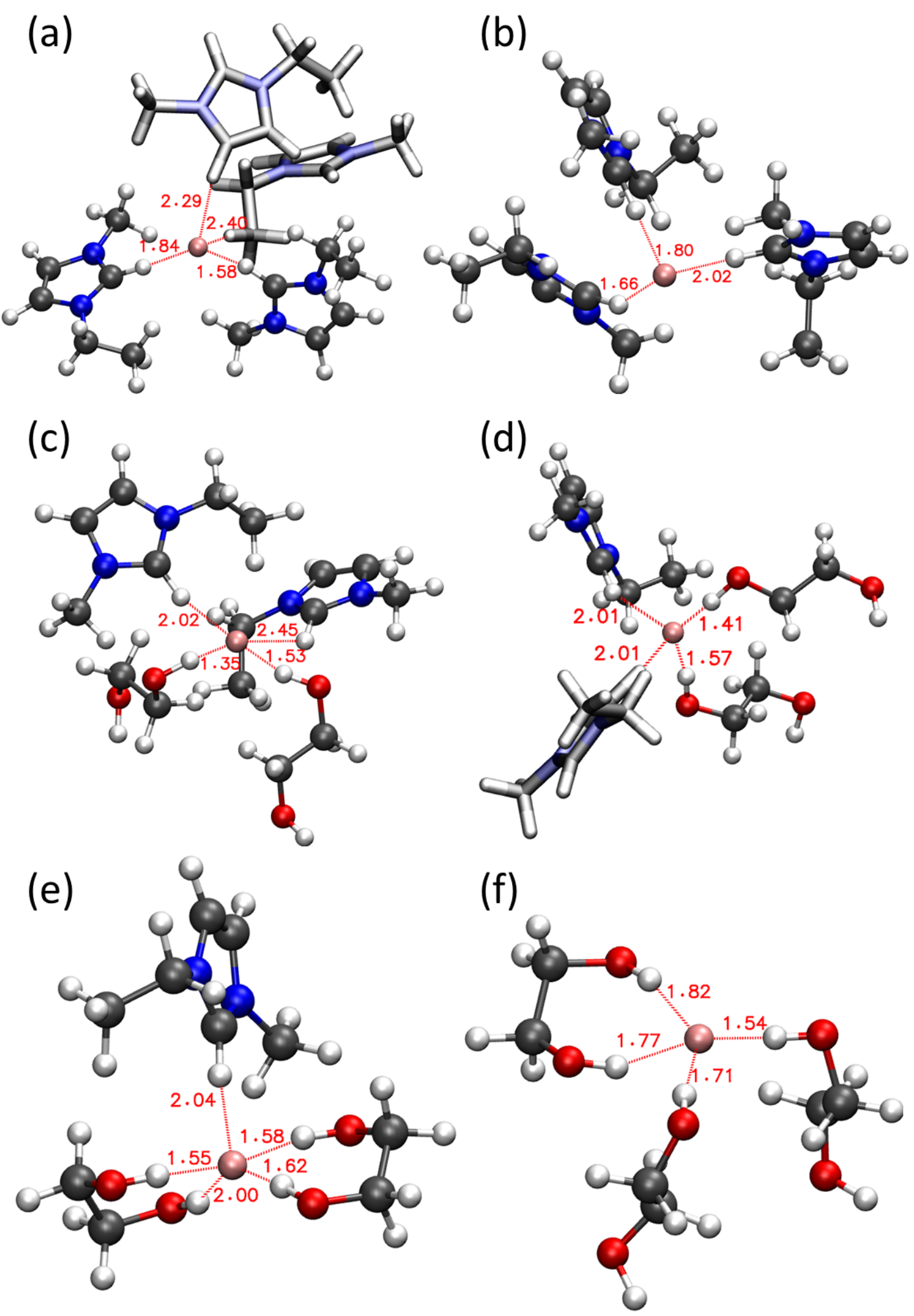

Figure S11: Coordination environment of some selected fluoride ions obtained from CP2KBOMD simulation of liquid [EMIM]F.0.5EG. Molecules, whose any atom if located within a distance of $2.5 \AA$ of the chosen fluoride ion, are shown. If fluoride is hydrogen bonded to $\mathrm{H}_{a}$ or $\mathrm{HO}$, the corresponding molecule is shown ${ }^{\mathrm{S}} \mathrm{g}$ ball and stick; otherwise, atoms and bonds are represented as cylinders. Distances are in $\AA$. Color code: hydrogen, white; carbon, gray; nitrogen, blue; oxygen, red; fluorine, salmon. 
S9 EG-F hydrogen bond network analysis
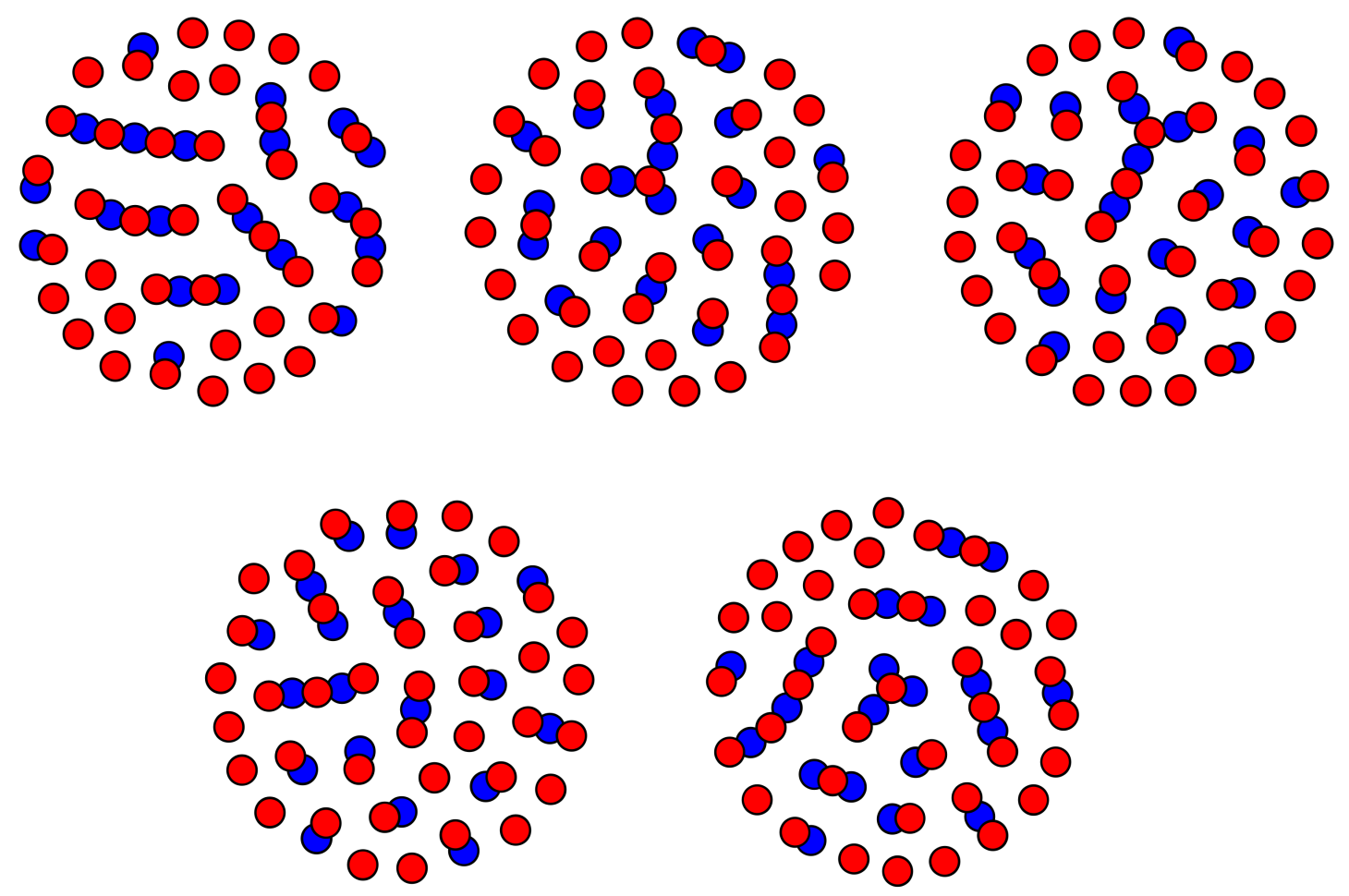

Figure S12: Network representation of the [EMIM]F.0.5EG liquid constructed from the configurations post CP2K-AIGO. Color scheme: red-F ; blue-EG. 

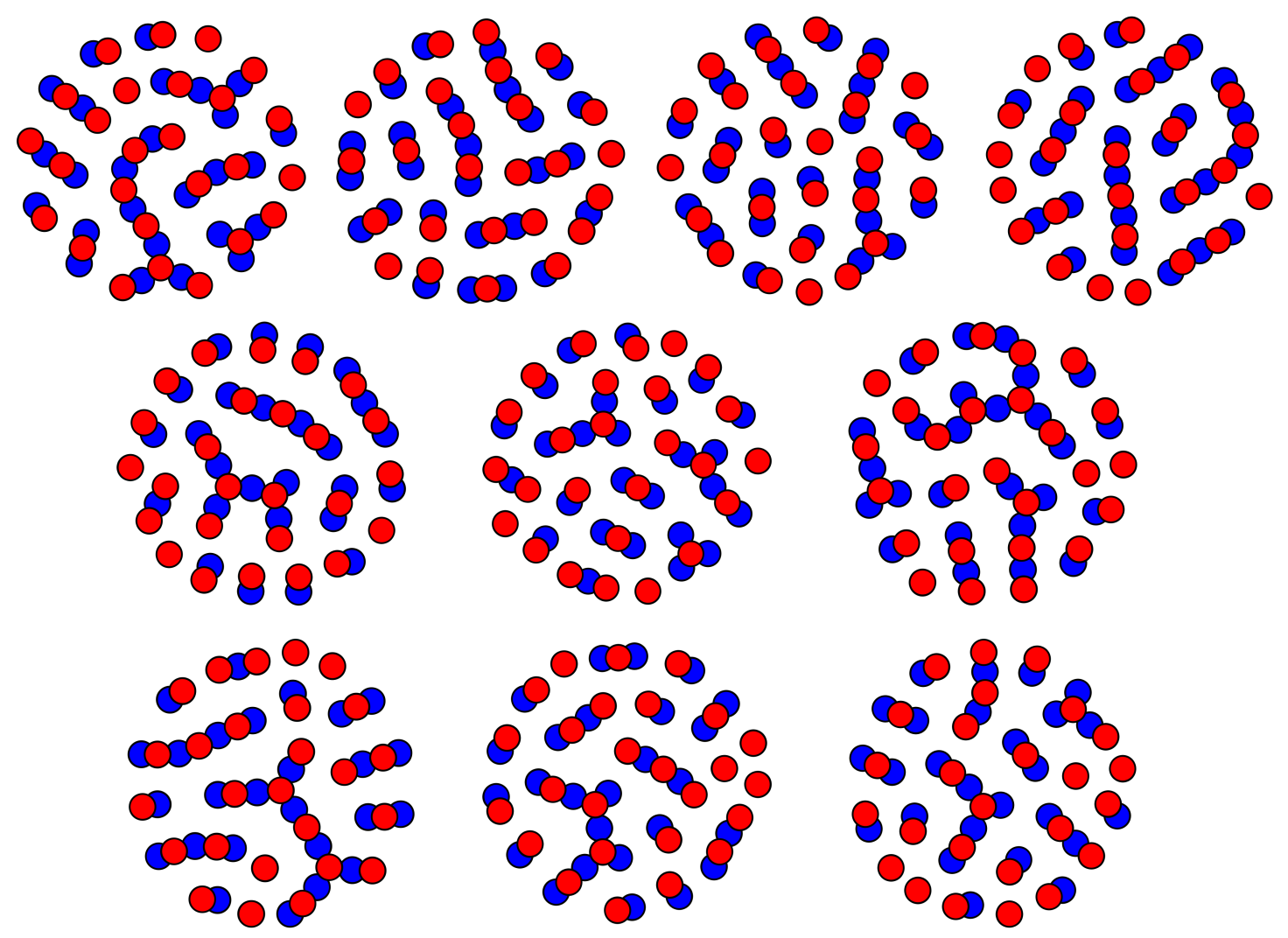

Figure S13: Network representation of the [EMIM]F.EG liquid constructed from the configurations post CP2K-AIGO. Color scheme: red-F ; blue-EG. 

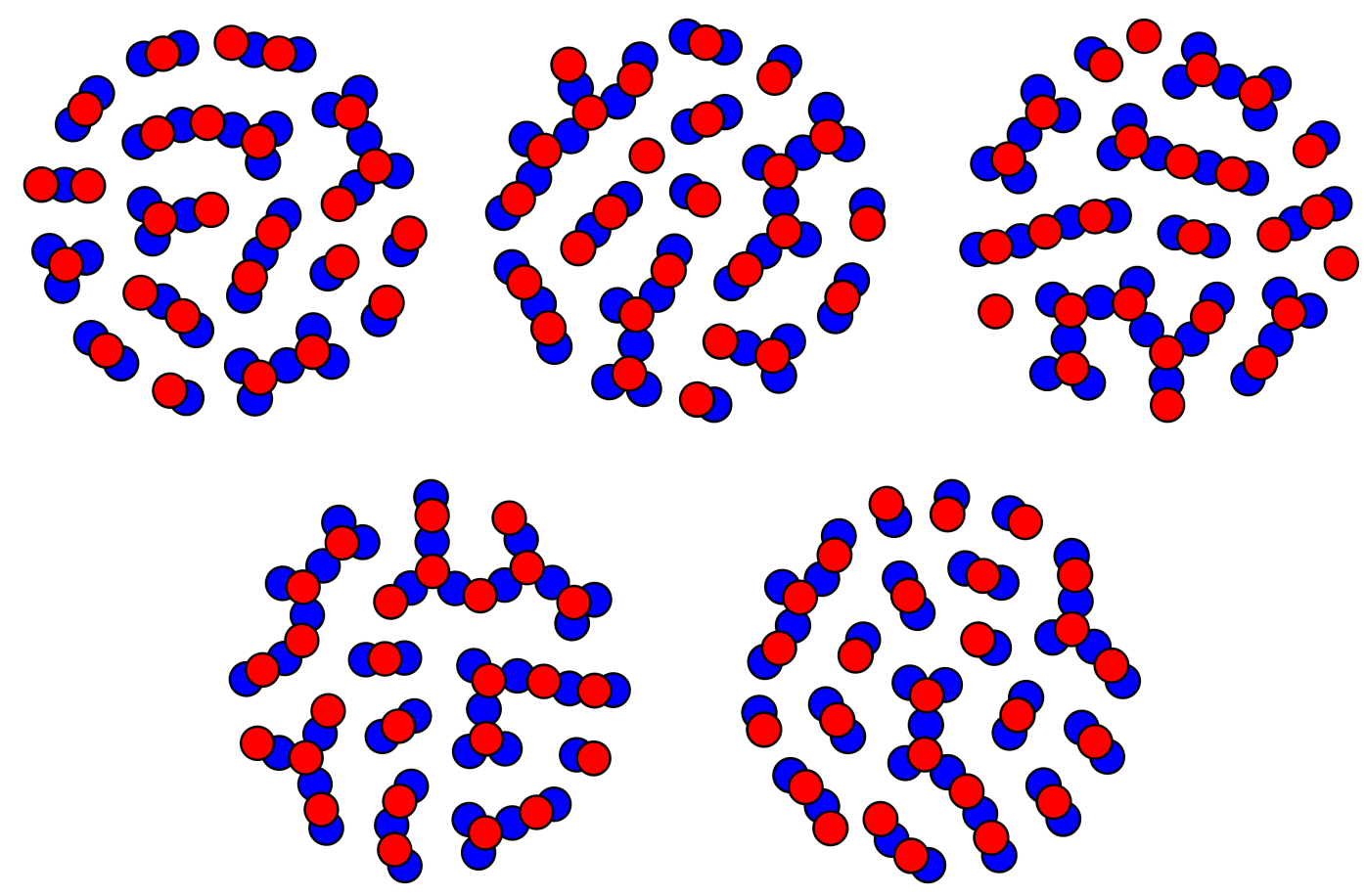

Figure S14: Network representation of the [EMIM]F.1.5EG liquid constructed from the configurations post CP2K-AIGO. Color scheme: red-F ; blue-EG.

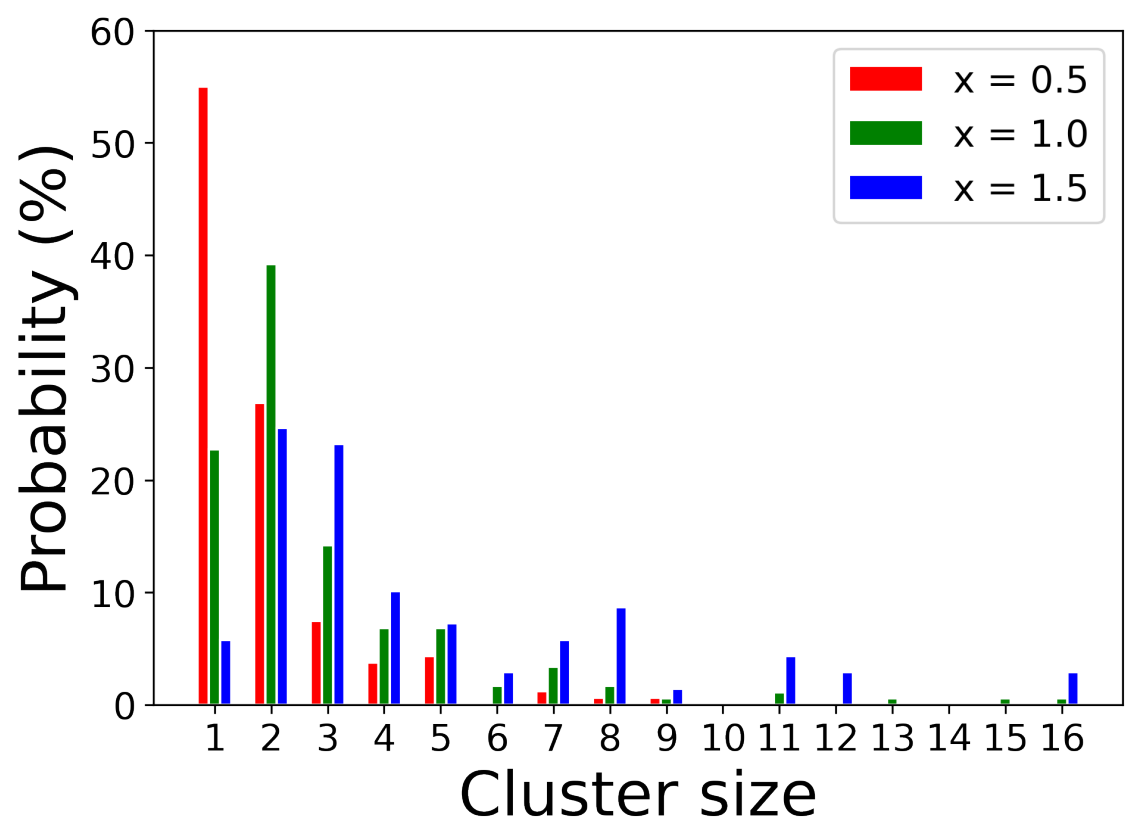

Figure S15: Percentage of clusters with the size of the clusters in [EMIM]F.xEG ( $\mathrm{x}=0.5,1.0$, 1.5), obtained from CP2K-AIGO via network analysis. 


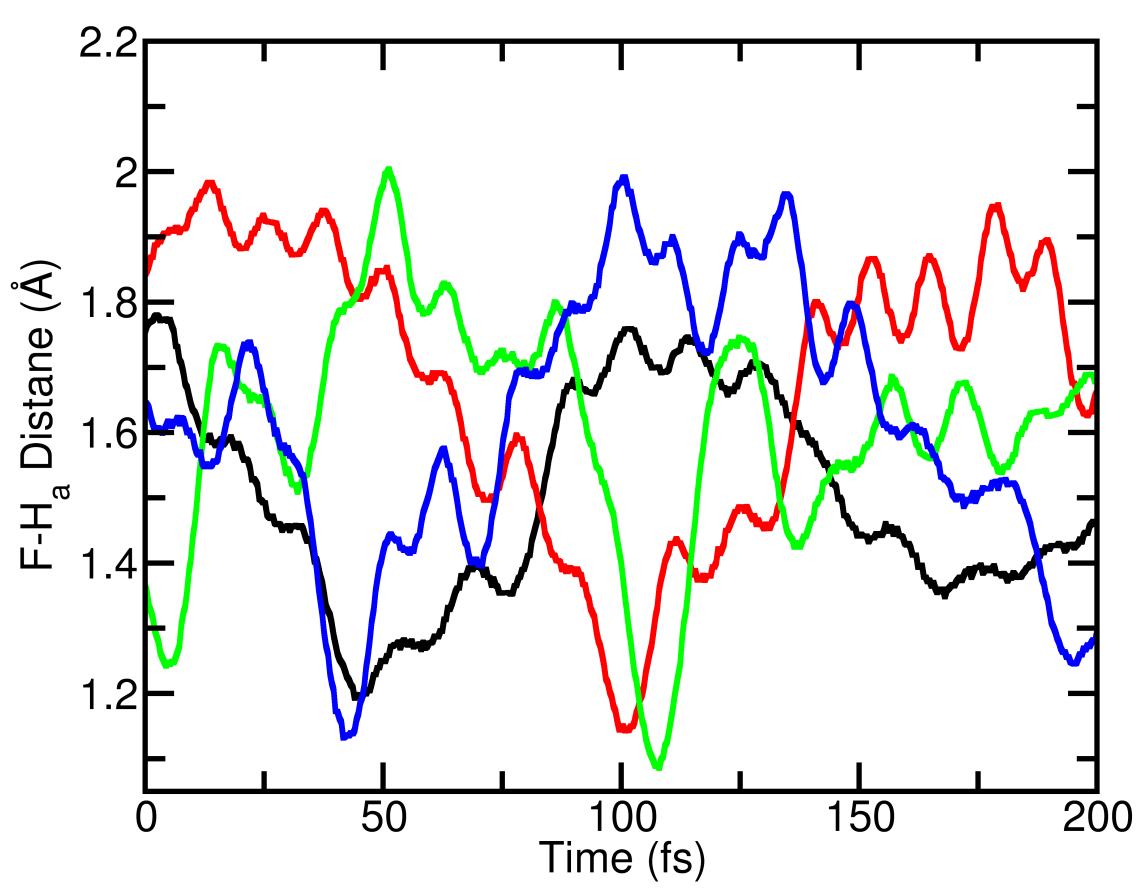

Figure S16: Some selected $\mathrm{F}-\mathrm{H}_{a}$ distances w.r.t. the course of simulation time in [EMIM]F. Within $200 \mathrm{fs}$, the HF distances reduce to $1.1 \AA$.

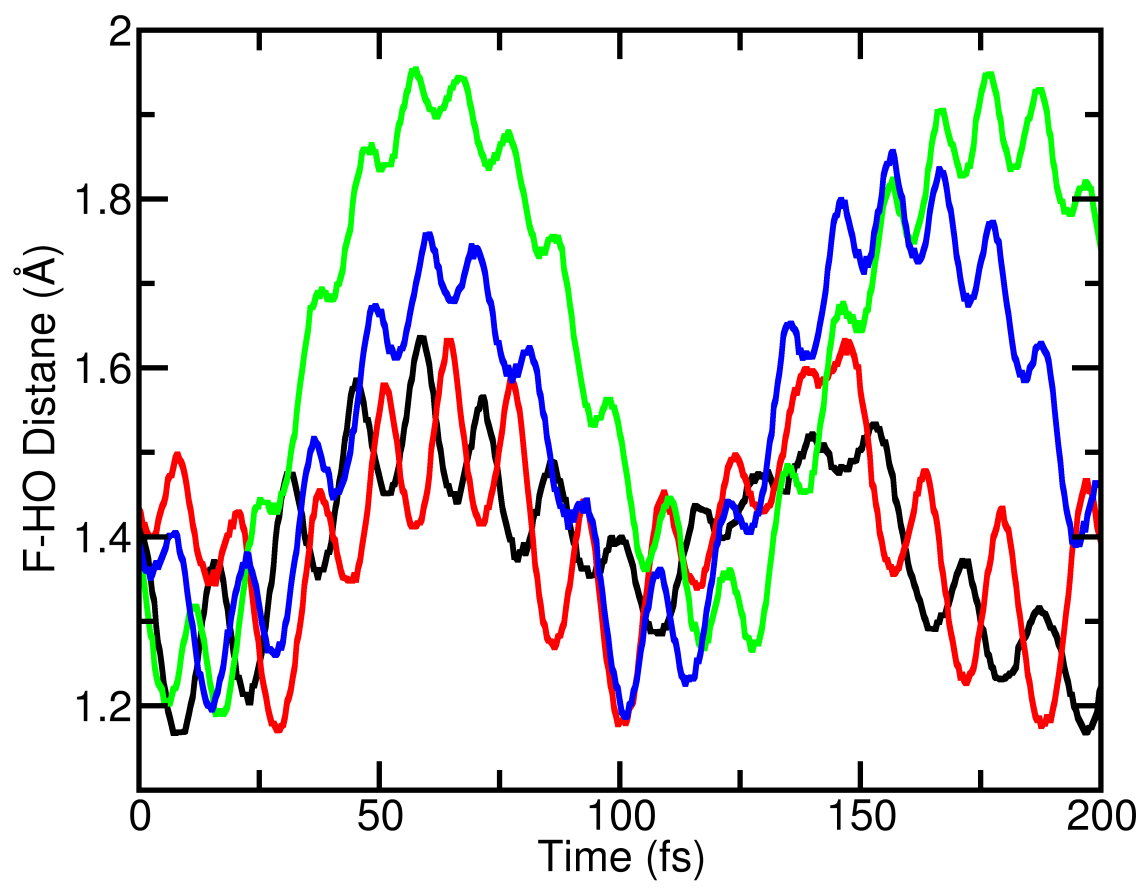

Figure S17: Some selected F-HO distances w.r.t. the course of simulation time in [EMIM]F.0.5EG system. 


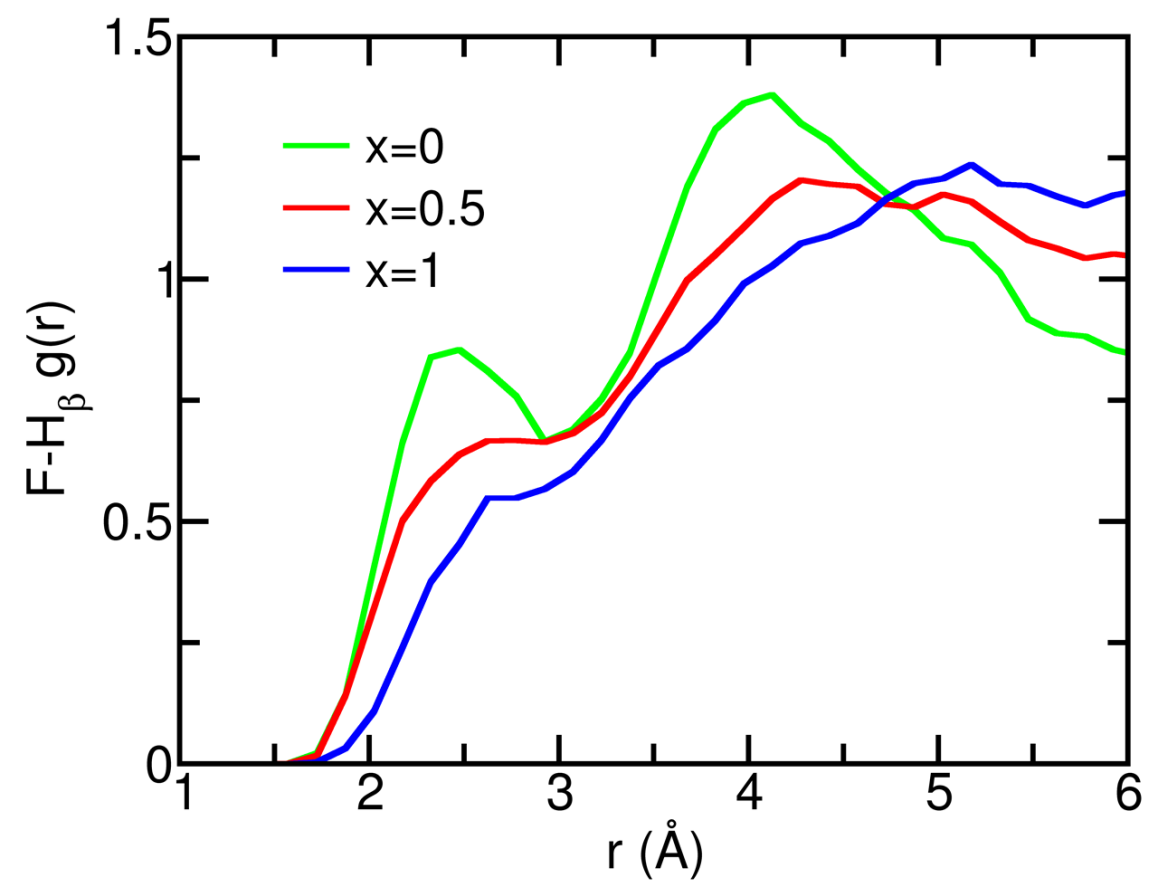

Figure S18: $\mathrm{F}-\mathrm{H}_{\beta}$ pair correlation functions in liquid [EMIM]F, [EMIM]F.0.5EG and [EMIM]F.EG, at 300K obtained from CP2K-BOMD simulations. 


\section{References}

(1) Mierzwicki, K.; Latajka, Z. Basis set superposition error in N-body clusters. Chem. Phys. Lett 2003, 380, $654-664$.

(2) Plumley, J. A.; Dannenberg, J. J. A comparison of the behavior of functional/basis set combinations for hydrogen-bonding in the water dimer with emphasis on basis set superposition error. J. Comput. Chem. 2011, 32, 1519-1527.

(3) Matthews, R. P.; Welton, T.; Hunt, P. A. Competitive pi interactions and hydrogen bonding within imidazolium ionic liquids. Phys. Chem. Chem. Phys. 2014, 16, 32383253.

(4) Wells, B. H.; Wilson, S. van der Waals interaction potentials: Many-body basis set superposition effects. Chemical Physics Letters 1983, 101, 429 - 434.

(5) Hostaš, J.; Řezáč, J. Accurate DFT-D3 Calculations in a Small Basis Set. J. Chem. Theory Comput 2017, 13, 3575-3585, PMID: 28715628.

(6) Hassanali, A. A.; Zhong, D.; Singer, S. J. An AIMD Study of the CPD Repair Mechanism in Water: Reaction Free Energy Surface and Mechanistic Implications. The J. of Phys. Chem. B 2011, 115, 3848-3859, PMID: 21417374.

(7) Piccinin, S.; Fabris, S. A first principles study of water oxidation catalyzed by a tetraruthenium-oxo core embedded in polyoxometalate ligands. Phys. Chem. Chem. Phys. 2011, 13, 7666-7674.

(8) Perlt, E.; Ray, P.; Hansen, A.; Malberg, F.; Grimme, S.; Kirchner, B. Finding the best density functional approximation to describe interaction energies and structures of ionic liquids in molecular dynamics studies. The Journal of Chemical Physics 2018, $148,193835$. 
(9) M.J. Abraham, E. L. B. H., D. van der Spoel; the GROMACS development team, GROMACS User Manual version 2018.

(10) Martinez, L.; Andrade, R.; Birgin, E. G.; Martinez, J. M. Packmol: A Package for Building Initial Configurations forMolecular Dynamics Simulations. J. Comput. Chem. 2009, 30, 2157-2164.

(11) Kelkar, M. S.; Shi, W.; Maginn, E. J. Determining the Accuracy of Classical Force Fields for Ionic Liquids: Atomistic Simulation of the Thermodynamic and Transport Properties of 1-Ethyl-3-methylimidazolium Ethylsulfate ([emim][EtSO4]) and Its Mixtures with Water. Indus. Eng. Chem. Res. 2008, 47, 9115-9126.

(12) Kaur, S.; Shobhna,; Kashyap, H. K. Insights Gained from Refined Force-Field for Pure and Aqueous Ethylene Glycol through Molecular Dynamics Simulations. J. Phys. Chem. B 2019, 123, 6543-6553, PMID: 31335141.

(13) Ponder, J. W.; Case, D. A. Protein Simulations; Advances in Protein Chemistry; Academic Press, 2003; Vol. 66; pp $27-85$.

(14) Nocedal, J.; Wright, S. J. Numerical Optimization; Springer Series in Operations Research, 1999.

(15) Nosé, S. A unified formulation of the constant temperature molecular dynamics methods. J. Chem. Phys. 1984, 81, 511-519.

(16) Nosé, S. A molecular dynamics method for simulations in the canonical ensemble. Mol. Phys. 1984, 52, 255-268.

(17) Parrinello, M.; Rahman, A. Polymorphic transitions in single crystals: A new molecular dynamics method. J. App. Phys. 1981, 52, 7182-7190.

(18) Chen, Z.; Tonouchi, Y.; Matsumoto, K.; Saimura, M.; Atkin, R.; Nagata, T.; 
Katahira, M.; Hagiwara, R. Partially Naked Fluoride in Solvate Ionic Liquids. J. Phys. Chem. Lett. 2018, 9, 6662-6667.

(19) Adamo, C.; Barone, V. Toward reliable density functional methods without adjustable parameters: The PBE0 model. J. Chem. Phys. 1999, 110, 6158-6170.

(20) Sabatini, R.; Gorni, T.; de Gironcoli, S. Nonlocal van der Waals density functional made simple and efficient. Phys. Rev. B 2013, 87, 041108.

(21) Fortes, A. D.; Suard, E. Crystal structures of ethylene glycol and ethylene glycol monohydrate. J. Chem. Phys. 2011, 135, 234501.

(22) Jindal, A.; Vasudevan, S. Conformation of Ethylene Glycol in the Liquid State: Intraversus Intermolecular Interactions. J. Phys. Chem. B 2017, 121, 5595-5600, PMID: 28490176.

(23) Kazerouni, M. R.; Hedberg, L.; Hedberg, K. Conformational Analysis. 21. Ethane1,2-diol. An Electron-Diffraction Investigation, Augmented by Rotational Constants and ab Initio Calculations, of the Molecular Structure, Conformational Composition, SQM Vibrational Force Field, and Anti-Gauche Energy Difference with Implications for Internal Hydrogen Bonding. J. Am. Chem. Soc. 1997, 119, 8324-8331.

(24) Christen, D.; Coudert, L.; Larsson, J.; Cremer, D. The RotationalTorsional Spectrum of the gGg Conformer of Ethylene Glycol: Elucidation of an Unusual Tunneling Path. J. Mol. Spec. 2001, 205, $185-196$.

(25) Das, P.; Das, P. K.; Arunan, E. Conformational Stability and Intramolecular Hydrogen Bonding in 1,2-Ethanediol and 1,4-Butanediol. J. Phys. Chem. A 2015, 119, 3710-3720, PMID: 25839224.

(26) Chopra, D.; Row, T. N. G.; Arunan, E.; Klein, R. A. Crystalline ethane-1,2-diol does not 
have intra-molecular hydrogen bonding: Experimental and theoretical charge density studies. J. Mol. Struct. 2010, 964, $126-133$.

(27) Crittenden, D. L.; Thompson, K. C.; Jordan, M. J. T. On the Extent of Intramolecular Hydrogen Bonding in Gas-Phase and Hydrated 1,2-Ethanediol. J. Phys. Chem. A 2005, 109, 2971-2977, PMID: 16833617. 